\title{
A New Robust Nonfragile Controller Design Scheme for a Class of Hybrid Systems through Piecewise Affine Models
}

\author{
Yunsai Chen $\left(\mathbb{D},{ }^{1,2}\right.$ Yongjie Pang, ${ }^{1}$ Zhao Yang, ${ }^{3}$ and Liang $\mathrm{Ma}^{3}$ \\ ${ }^{1}$ College of Shipbuilding Engineering, Harbin Engineering University, Harbin 150001, China \\ ${ }^{2}$ Department of Technology, National Deep Sea Center, Qingdao 266237, China \\ ${ }^{3}$ Qingdao National Laboratory for Marine Science and Technology, Scientific and Technology Infrastructure Department, \\ Qingdao 266237, China
}

Correspondence should be addressed to Yunsai Chen; cys@ndsc.org.cn

Received 19 July 2018; Revised 26 September 2018; Accepted 27 September 2018; Published 21 October 2018

Academic Editor: Leonardo Acho

Copyright (C) 2018 Yunsai Chen et al. This is an open access article distributed under the Creative Commons Attribution License, which permits unrestricted use, distribution, and reproduction in any medium, provided the original work is properly cited.

\begin{abstract}
This paper investigates the robust $\mathscr{H}_{\infty}$ nonfragile control problem for a class of discrete-time hybrid systems based on piecewise affine models. The objective is to develop an admissible piecewise affine nonfragile controller such that the resulting closed-loop system is asymptotically stable with robust $\mathscr{H}_{\infty}$ performance $\gamma$. By employing a state-control augmentation methodology, some new sufficient conditions for the controller synthesis are formulated based on piecewise Lyapunov functions (PLFs). The controller gains can be obtained via solving a set of linear matrix inequalities. Simulation examples are finally presented to demonstrate the feasibility and effectiveness of the proposed approaches.
\end{abstract}

\section{Introduction}

Over the past few decades, hybrid systems have drawn tremendous attention from the control community, as they contain the competence to model the interaction between logic components and continuous dynamics [1-6]. Piecewise affine (PWA) systems are a rich class of hybrid systems, which can provide a useful modeling approach for the stability analysis and controller synthesis of hybrid systems [7-9]. In addition, the PWA models can approximate smooth nonlinear plants with arbitrary degrees of accuracy $[10,11]$.

In recent years, many valuable references on the systematic analysis and synthesis for the PWA systems have been published [12-22]. In [12], the stability of continuous-time PWA systems was analyzed by utilizing discontinuous piecewise Lyapunov functions (PLFs). On the basis of the idea in [12], some algorithms for stability and performance analysis of discrete-time PWA systems were proposed in [14] and the continuity of the PLFs was not required for the discrete-time case. The authors in [20] designed a static output-feedback controller for discrete-time PWA systems such that the resulting closed-loop system was asymptotically stable with robust $\mathscr{H}_{\infty}$ performance $\gamma$. The authors in [21] developed a reconfigurable control approach for continuous-time PWA systems with actuator and sensor faults, and the output tracking problem was investigated simultaneously. In [23], the problem of delay-dependent fixed-order memory piecewise affine $\mathscr{H}_{\infty}$ output-feedback control for a class of nonlinear systems with time-varying delay was considered. The authors in [24] have proposed a novel system-augmentation approach to the delay-dependent reliable piecewise affine $\mathscr{H}_{\infty}$ static output-feedback control for nonlinear systems with timevarying delay and sensor faults in the piecewise-MarkovianLyapunov-functional-based framework.

On the other hand, since 1980s, the robust $\mathscr{H}_{\infty}$ control problem has drawn much attention as it is robust against the system parameter uncertainties, unmodeled dynamics, and external disturbances $[25,26]$. It is implicitly assumed in this control approach that the controllers are implemented precisely. In fact, controllers gains inevitably possess some degrees of errors or parameter variations due to physical limitations such as the inherent inaccuracy in analog systems, finite word length in arbitrary digital systems, and round-off errors in process of numerical computations [27]. 
Numbers of literatures have already shown that relatively small perturbations in the controller parameters can even result in instability of the closed-loop system [28-30]. For this case, it is realistic to synthesize a controller for a given uncertain system such that the controller is insensitive to some predefined admissible variations with respect to its gains. More recently, many efforts have been devoted to the nonfragile robust $\mathscr{H}_{\infty}$ controller design problem [31-40]. For instance, a nonfragile $\mathscr{H}_{\infty}$ controller with multiplicative gain perturbations was proposed for linear time invariant (LTI) systems in [31]. The author in [32] developed a decentralized robust state-feedback nonfragile control method for uncertain discrete-time large-scale systems with time-delays and controller gain variations. In [34], a nonfragile robust controller with parameter uncertainties was constructed for a class of nonlinear systems based on T-S fuzzy neural models. The authors in [40] considered the nonfragile robust $\mathscr{H}_{\infty}$ control problem for fuzzy systems based on output information. In spite of the attractive features in piecewise affine systems, the authors in [41] proposed a state-feedback nonfragile robust controller design method for continuoustime PWA systems. The results in [41] were derived based on a common Lyapunov function (CLF), and the matrices $B_{i}$ are not empowered to have uncertainties. To the best of the authors' knowledge, few attempts have been devoted to the robust nonfragile controller design for discrete-time piecewise affine systems based on PLFs, which inspires us for this study.

In this paper, we will consider the piecewise affine nonfragile control problem for a class of discrete-time hybrid systems based on piecewise affine models. Via employing a state-control augmentation approach, a new nonfragile controller synthesis method is proposed based on piecewise Lyapunov functions (PLFs), which removes the restrictive condition that the input matrices do not have uncertainties. This feature enables one to design the nonfragile controller for a broader class of industrial systems. It is shown that the resulting closed-loop PWA system is asymptotically stable with a prescribed disturbance attenuation level $\gamma$, and the controller gains can be acquired via solving a set of linear matrix inequalities (LMIs). Finally, two simulation examples are presented to show the effectiveness and amenability of the proposed approaches. The main contributions of this paper are listed as follows: (1) The controller is designed based on PLFs and piecewise affine models and the conservation can be reduced. (2) A new robust piecewise affine nonfragile controller is designed to deal with the situations that perturbations exist in the controller gains. (3) The input matrices are empowered to have uncertainties, which is more applicable for some practical requirements.

The rest of this paper is organized as follows. The piecewise affine model description and nonfragile controller design are given in Section 2 . The asymptotic stability analysis for resulting closed-loop system is shown in Section 3. Simulation examples are shown in Section 4 to verify the effectiveness of the proposed methods. Section 5 gives the conclusions.
Notations. The notations employed in this paper are standard. A real symmetric matrix $A>0(\geq 0)$ indicates $A$ being positive-definite (positive-semi-definite). $\operatorname{sym}\{X\}$ is the shorthand notation for $X^{\mathrm{T}}+X$.

\section{Problem Formulation}

2.1. Discrete-Time Piecewise Affine Systems. A discrete-time piecewise affine system is shown as

$$
\begin{aligned}
x(t+1)= & \left(A_{i}+\Delta A_{i}\right) x(t)+a_{i}+\Delta a_{i} \\
& +\left(B_{i}+\Delta B_{i}\right) u(t)+H_{i} w(t) \\
z(t)=L_{i} x(t)+N_{i} u(t), & \\
& x(t) \in \Sigma_{i}, \quad i \in \Pi:=\{1,2, \ldots, \phi\}
\end{aligned}
$$

where $x(t) \in \mathfrak{R}^{n}$ is the system state; $u(t) \in \mathfrak{R}^{m}$ is the control input; $z(t) \in \mathfrak{R}^{p}$ is the regulated output; $w(t)$ is the external nonlinear disturbance belonging to $l_{2}[0, \infty) ; \Sigma_{i}$ is a polyhedral partition from the state-space and $\Pi$ is the index set of these polyhedral regions; $\Delta A_{i}, \Delta a_{i}$, and $\Delta B_{i}$ are the uncertainty terms of the $l$-th local affine model satisfying

$$
\left[\begin{array}{lll}
\Delta A_{i} & \Delta a_{i} & \Delta B_{i}
\end{array}\right]=W_{i 1} \Delta_{i}(t)\left[\begin{array}{lll}
M_{i 1} & M_{i 2} & M_{i 3}
\end{array}\right], \quad i \in \Pi
$$

with $W_{i 1}, M_{i 1}, M_{i 2}$, and $M_{i 3}$ being known real constant matrices. $\Delta_{i}(t)$ is unknown time-varying matrix satisfying

$$
\Delta_{i}^{\mathrm{T}}(t) \Delta_{i}(t) \leq \mathbf{I}
$$

Remark 1. The system model in (1) is in fact affine systems rather than linear systems as the offset term $\left(a_{i}+\Delta a_{i}\right)$ is involved. One can easily conclude that this type of models is more accurate for approximation to nonlinear systems [41].

Following the idea given in [20], define the region index $\Pi=\Pi_{0} \cup \Pi_{1}$, where $\Pi_{1}$ denotes the index set of subspaces without the origin and $\Pi_{0}$ refers to the index set of subspaces containing the origin.

For future use, a new set $\Omega$ is introduced to depict all possible region transitions

$$
\Omega:=\left\{(i, j) \mid x(t) \in \Sigma_{i}, x(t+1) \in \Sigma_{j}, i, j \in \Pi\right\} .
$$

When $(i, j) \in \Omega$ and $i=j$, the state is involved in the same region $\Sigma_{i}$ at the time $t$. Otherwise, the state trajectories will jump from the region $\Sigma_{i}$ to $\Sigma_{j}$ at that time.

In this paper, it is assumed that each polyhedral regions $\Sigma_{i}$ can be outer approximated by an ellipsoid $\Psi_{i}$, that is to say, there exist matrices $Q_{i}$ and $q_{i}$ such that

$$
\Sigma_{i} \subseteq \Psi_{i}, \quad \Psi_{i}:=\left\{x \mid\left\|Q_{i} x+q_{i}\right\| \leq 1\right\} .
$$

When $\Sigma_{i}$ are slab regions, the above covering is useful, and then the parameters $Q_{i}$ and $q_{i}$ are guaranteed to exist, and $\Sigma_{i} \subseteq \Psi_{i}$ and $\Psi_{i} \subseteq \Sigma_{i}$. If the polyhedral regions $\Sigma_{i}$ are slabs with

$$
\Sigma_{i}=\left\{x \mid \alpha_{i} \leq x \leq \beta_{i}\right\}, \quad i \in \Pi
$$


where $\alpha_{i}, \beta_{i} \in \mathfrak{R}, \phi_{i} \in \mathfrak{R}^{n}$, then each slab can be represented by a degenerate ellipsoid as in (6) with

$$
\begin{aligned}
Q_{i} & =\frac{2 \phi_{i}^{\mathrm{T}}}{\beta_{i}-\alpha_{i}}, \\
q_{i} & =\frac{\beta_{i}+\alpha_{i}}{\alpha_{i}-\beta_{i}} .
\end{aligned}
$$
ship:

For each ellipsoid region, we have the following relation-

$$
\left[\begin{array}{c}
1 \\
x(t)
\end{array}\right]^{\mathrm{T}}\left[\begin{array}{cc}
q_{i}^{\mathrm{T}} q_{i}-1 & q_{i}^{\mathrm{T}} Q_{i} \\
\star & Q_{i}^{\mathrm{T}} Q_{i}
\end{array}\right]\left[\begin{array}{c}
1 \\
x(t)
\end{array}\right] \leq 0, \quad i \in \Pi .
$$

2.2. Piecewise Affine Nonfragile Controller Design. Borrowing the idea from [42], define $\xi(t)=\left[\begin{array}{lll}x^{\mathrm{T}}(t) & u^{\mathrm{T}}(t)\end{array}\right]^{\mathrm{T}}$. As the inaccuracies or uncertainties inevitably involved in the controllers in many practical situations, for the piecewise affine system (1), a new nonfragile piecewise affine controller in differential form is designed as

$$
u(t+1)=\left(K_{i}+\Delta K_{i}\right) \xi(t)+k_{i}+\Delta k_{i},
$$

where $K_{i} \in \mathfrak{R}^{n_{u} \times\left(n_{x}+n_{u}\right)}$ and $k_{i} \in \mathfrak{R}^{n_{u}}$ are the controller gains to be determined, and $k_{i}=0$ for $i \in \Pi_{0}$. $\Delta K_{i}$ and $\Delta k_{i}$ are unknown matrices standing for the gain perturbations satisfying

$$
\left[\begin{array}{ll}
\Delta K_{i} & \Delta k_{i}
\end{array}\right]=W_{i 2} \Delta_{u i}(t)\left[\begin{array}{ll}
U_{i 1} & U_{i 2}
\end{array}\right], \quad i \in \Pi,
$$

where $W_{i 2}, U_{i 1}$, and $U_{i 2}$ are known real constant matrices of appropriate dimensions. The real-valued matrix functions $\Delta_{u i}(t)$ satisfy

$$
\Delta_{u i}^{\mathrm{T}}(t) \Delta_{u i}(t) \leq \mathbf{I}
$$

The parameter uncertainties in (1) and (9) are recognized to be admissible if (2)-(3) and (10)-(11) hold.

By applying the piecewise affine nonfragile controller (9) into system (1), the closed-loop system can be formulated as

$$
\begin{aligned}
\xi(t+1) & =\left(\mathscr{A}_{i}+\Delta \mathscr{A}_{i}\right) \xi(t)+\mathrm{a}_{i}+\Delta \mathrm{a}_{i}+\bar{H}_{i} w(t) \\
z(t) & =\bar{L}_{i} \xi(t), \\
x(t) & \in \Sigma_{i},
\end{aligned}
$$

$i \in \Pi$,

where $\mathscr{A}_{i}=\bar{A}_{i}+R K_{i}, \Delta \mathscr{A}_{i}=\Delta \bar{A}_{i}+R \Delta K_{i}, \mathrm{a}_{i}=\bar{a}_{i}+R k_{i}$, $\Delta \mathrm{a}_{i}=\Delta \bar{a}_{i}+R \Delta k_{i}$, and

$$
\begin{aligned}
\bar{A}_{i} & =\left[\begin{array}{cc}
A_{i} & B_{i} \\
\mathbf{0} & \mathbf{0}
\end{array}\right], \\
\Delta \bar{A}_{i} & =\left[\begin{array}{cc}
\Delta A_{i} & \Delta B_{i} \\
\mathbf{0} & \mathbf{0}
\end{array}\right],
\end{aligned}
$$

$$
\begin{aligned}
\bar{a}_{i} & =\left[\begin{array}{c}
a_{i} \\
\mathbf{0}
\end{array}\right], \\
\Delta \bar{a}_{i} & =\left[\begin{array}{c}
\Delta a_{i} \\
\mathbf{0}
\end{array}\right], \\
R & =\left[\begin{array}{c}
\mathbf{0} \\
\mathbf{I}_{n_{u}}
\end{array}\right], \\
\bar{H}_{i} & =\left[\begin{array}{c}
H_{i} \\
\mathbf{0}
\end{array}\right], \\
\bar{L}_{i} & =\left[\begin{array}{ll}
L_{i} & N_{i}
\end{array}\right] .
\end{aligned}
$$

Based on the augment vector $\xi(t)$, the following inequality implies (8) for each ellipsoid region,

$$
\left[\begin{array}{c}
1 \\
\xi(t)
\end{array}\right]^{\mathrm{T}}\left[\begin{array}{rr}
q_{i}^{\mathrm{T}} q_{i}-1 & q_{i}^{\mathrm{T}} \overline{\mathrm{Q}}_{i} \\
\star & \overline{\mathrm{Q}}_{i}^{\mathrm{T}} \bar{Q}_{i}
\end{array}\right]\left[\begin{array}{c}
1 \\
\xi(t)
\end{array}\right] \leq 0, \quad i \in \Pi,
$$

where $\bar{Q}_{\mathrm{i}}=\left[\begin{array}{ll}Q_{i} & \mathbf{0}_{n_{u} \times n_{u}}\end{array}\right]$.

As practical control systems are always subject to external disturbance, thus, in this paper, we aim to synthesize an admissible piecewise affine nonfragile controller in the form of (9) such that the resulting closed-loop system is asymptotically stable with robust $\mathscr{H}_{\infty}$ performance $\gamma$ as

$$
\|z\|_{2}<\gamma\|w\|_{2}
$$

under zero initial conditions for all nonzero $w(t) \in l_{2}[0, \infty]$.

Before ending this section, the following lemmas will be employed to prove the main results in this paper.

Lemma 2 (see [22]). Real matrices $Q>0, R, \Delta(t)$, and $S$ are regular matrices with appropriate dimensions. $\Delta(t)$ is timevarying satisfying $\Delta^{\mathrm{T}}(t) \Delta(t) \leq \mathbf{I}$. The inequality

$$
Q+R \Delta(t) S+S^{\mathrm{T}} \Delta^{\mathrm{T}}(t) R^{\mathrm{T}}<0
$$

holds if and only if for some $\varepsilon>0$

$$
Q+\varepsilon R^{\mathrm{T}} R+\varepsilon^{-1} S^{\mathrm{T}} S<0 .
$$

\section{Piecewise Affine Nonfragile Controller Analysis and Design}

On the basis of piecewise Lyapunov functions (PLFs), some new approaches to robust $\mathscr{H}_{\infty}$ piecewise affine nonfragile controller synthesis will be proposed in this section. 
Theorem 3. For a given constant scalar $\lambda$, the closed-loop system (12) is asymptotically stable with disturbance attenuation level $\gamma$ if there exist matrices $0<P_{i}=P_{i}^{\mathrm{T}} \in \mathfrak{R}^{(n+m) \times(n+m)}, \bar{K}_{i} \in$ $\mathfrak{R}^{m \times(n+m)}, \bar{k}_{i} \in \mathfrak{R}^{m}, V_{i 1} \in \mathfrak{R}^{n \times n}, V_{i 2} \in \mathfrak{R}^{m \times n}, V_{i 3} \in \mathfrak{R}^{m \times m}$, and $i \in \Pi$ and two sets of scalars $\left\{r_{i j}<0, i \in \Pi_{1},(i, j) \in \Omega\right\}$, and $\left\{\varepsilon_{i}>0, i \in \Pi\right\}$, such that the following LMIs hold:

$$
\begin{gathered}
{\left[\begin{array}{ccc}
\Gamma_{i j 1}+\varepsilon_{i j} \Theta_{i 1}^{\mathrm{T}} \Theta_{i 1} & \Lambda_{i 1}^{(1)} & \Lambda_{i 1}^{(2)} \\
\star & -\varepsilon_{i j} \mathbf{I} & \mathbf{0} \\
\star & \star & -\varepsilon_{i j} \mathbf{I}
\end{array}\right]<0,} \\
\quad i \in \Pi_{0}, \quad j \in \Pi, \quad(i, j) \in \Omega, \\
{\left[\begin{array}{ccc}
\Gamma_{i j 2}+\varepsilon_{i j} \Theta_{i 2}^{\mathrm{T}} \Theta_{i 2} & \Lambda_{i 2}^{(1)} & \Lambda_{i 2}^{(2)} \\
\star & -\varepsilon_{i j} \mathbf{I} & \mathbf{0} \\
\star & \star & -\varepsilon_{i j} \mathbf{I}
\end{array}\right]<0,} \\
\\
\end{gathered}
$$

where

$$
\begin{aligned}
& \Gamma_{i j 1}=\left[\begin{array}{ccc}
P_{j}-V_{i}-V_{i}^{\mathrm{T}} & V_{i} \bar{A}_{i}+E \bar{K}_{i} & V_{i} \bar{H}_{i} \\
\star & -P_{i}+\bar{L}_{i}^{\mathrm{T}} \bar{L}_{i} & \mathbf{0} \\
\star & \star & -\gamma^{2} \mathbf{I}
\end{array}\right], \\
& \Theta_{i 1}=\left[\begin{array}{cc}
\mathbf{0} & \mathbf{0} \\
\bar{M}_{i 1}^{\mathrm{T}} & U_{i 1}^{\mathrm{T}} \\
\mathbf{0} & \mathbf{0}
\end{array}\right], \\
& \Lambda_{i 1}^{(1)}=\left[\begin{array}{c}
V_{i} \bar{W}_{i 1} \\
\mathbf{0} \\
\mathbf{0}
\end{array}\right], \\
& \Lambda_{i 1}^{(2)}=\left[\begin{array}{c}
V_{i} R W_{i 2} \\
\mathbf{0} \\
\mathbf{0}
\end{array}\right],
\end{aligned}
$$$$
\Gamma_{i j 2}
$$$$
=\left[\begin{array}{cccc}
P_{j}-V_{i}-V_{\mathrm{i}}^{\mathrm{T}} & V_{i} \bar{a}_{i}+E \bar{k}_{i} & V_{i} \bar{A}_{i}+E \bar{K}_{i} & V_{i} \bar{H}_{i} \\
\star & -r_{i j}\left(1-q_{i}^{\mathrm{T}} q_{i}\right) & r_{i j} q_{i}^{\mathrm{T}} \overline{\mathrm{Q}}_{i} & 0 \\
\star & \star & -P_{i}+r_{i j} \overline{\mathrm{Q}}_{i}^{\mathrm{T}} \overline{\mathrm{Q}}_{i}+\bar{L}_{i}^{\mathrm{T}} \bar{L}_{i} & 0 \\
\star & \star & \star & -\gamma^{2} \mathrm{I}
\end{array}\right],
$$$$
\Theta_{i 2}=\left[\begin{array}{cc}
\mathbf{0} & \mathbf{0} \\
M_{i 2}^{\mathrm{T}} & U_{i 2}^{\mathrm{T}} \\
\bar{M}_{i 1}^{\mathrm{T}} & U_{i 1}^{\mathrm{T}} \\
\mathbf{0} & \mathbf{0}
\end{array}\right],
$$

$$
\begin{aligned}
& \Lambda_{i 2}^{(1)}=\left[\begin{array}{c}
V_{i} \bar{W}_{i 1} \\
\mathbf{0} \\
\mathbf{0} \\
\mathbf{0}
\end{array}\right], \\
& \Lambda_{i 2}^{(2)}=\left[\begin{array}{c}
V_{i} R W_{i 2} \\
\mathbf{0} \\
\mathbf{0} \\
\mathbf{0}
\end{array}\right], \\
& V_{i}=\left[\begin{array}{cc}
V_{i 1} & \lambda S V_{i 2} \\
V_{i 3} & V_{i 2}
\end{array}\right], \\
& E=\left[\begin{array}{c}
\lambda S \\
\mathbf{I}_{m}
\end{array}\right], \\
& \bar{A}_{i}=\left[\begin{array}{cc}
A_{i} & B_{i} \\
0 & 0
\end{array}\right], \\
& \bar{a}_{i}=\left[\begin{array}{c}
a_{i} \\
0
\end{array}\right], \\
& \bar{H}_{i}=\left[\begin{array}{c}
H_{i} \\
0
\end{array}\right], \\
& \bar{W}_{i 1}=\left[\begin{array}{c}
W_{i 1} \\
\mathbf{0}
\end{array}\right], \\
& \bar{M}_{i 1}=\left[\begin{array}{ll}
M_{i 1} & M_{i 3}
\end{array}\right],
\end{aligned}
$$

and $S \in \mathfrak{R}^{(n+m) \times m}$ is an arbitrary matrix.

Moreover, the controller gains for each subspace can be determined via

$$
\begin{aligned}
K_{i} & =V_{i 2}^{-1} \bar{K}_{i}, \\
k_{i} & =V_{i 2}^{-1} \bar{k}_{i} .
\end{aligned}
$$

Proof. It is easy to see that condition (18) for $i \in \Pi_{0}$ is a special case of condition (19) for $i \in \Pi_{1}$. Without loss of generality, in the following, the proof of the more complex case $i \in \Pi_{1}$ is to be considered. Take the piecewise Lyapunov function as

$$
V(t)=\xi^{\mathrm{T}}(t) P_{i} \xi(t), \quad i \in \Pi .
$$

It is also assumed that $x(t+1) \in \Sigma_{j}$. Then we have $V(t+$ $1)=\xi^{\mathrm{T}}(t+1) P_{j} \xi(t+1)$. The closed-loop system in (12) is asymptotically stable with robust $\mathscr{H}_{\infty}$ performance $\gamma$, if the inequality

$$
V(t+1)-V(t)+z^{\mathrm{T}}(t) z(t)-\gamma^{2} w^{\mathrm{T}}(t) w(t)<0
$$

holds.

By substituting (22) into (23), it yields that

$$
\begin{gathered}
\xi^{\mathrm{T}}(t+1) P_{j} \xi(t+1)-\xi^{\mathrm{T}}(t) P_{i} \xi(t)+z^{\mathrm{T}}(t) z(t) \\
-\gamma^{2} w^{\mathrm{T}}(t) w(t)<0, \quad(i, j) \in \Omega .
\end{gathered}
$$


Along the trajectories of closed-loop system (12), the following inequality implies $(24)$ with $(i, j) \in \Omega$,

$$
\begin{aligned}
& {\left[\begin{array}{c}
1 \\
\xi(t) \\
w(t)
\end{array}\right]^{\mathrm{T}}\left\{\left[\begin{array}{c}
\mathrm{a}_{i}^{\mathrm{T}}+\Delta \mathrm{a}_{i}^{\mathrm{T}} \\
\mathscr{A}_{i}^{\mathrm{T}}+\Delta \mathscr{A}_{i}^{\mathrm{T}} \\
\bar{H}_{i}^{\mathrm{T}}
\end{array}\right] P_{j}(\star)+\left[\begin{array}{c}
\mathbf{0} \\
\overline{\mathscr{L}}_{i}^{\mathrm{T}} \\
\mathbf{0}
\end{array}\right](\star)\right.} \\
& \left.+\left[\begin{array}{ccc}
\mathbf{0} & \mathbf{0} & \mathbf{0} \\
\star & -P_{i} & \mathbf{0} \\
\star & \star & -\gamma^{2} \mathbf{I}
\end{array}\right]\right\}\left[\begin{array}{c}
1 \\
\xi(t) \\
w(t)
\end{array}\right]<0 .
\end{aligned}
$$

Noticing the state-space partition (14) and utilizing Sprocedure, the following inequality implies (25) based on Schur complement:

$$
\begin{aligned}
& {\left[\begin{array}{cccc}
-P_{j}^{-1} & \mathrm{a}_{i}+\Delta \mathrm{a}_{i} & \mathscr{A}_{i}+\Delta \mathscr{A}_{i} & \bar{H}_{i} \\
\star & r_{i j}\left(q_{i}^{\mathrm{T}} q_{i}-1\right) & r_{i j} q_{i}^{\mathrm{T}} \bar{Q}_{i} & \mathbf{0} \\
\star & \star & -P_{i}+r_{i j} \bar{Q}_{i}^{\mathrm{T}} \bar{Q}_{i}+\bar{L}_{i}^{\mathrm{T}} \bar{L}_{i} & \mathbf{0} \\
\star & \star & \star & -\gamma^{2} \mathbf{I}
\end{array}\right]} \\
& <0, \quad i \in \Pi_{1}, \quad(i, j) \in \Omega .
\end{aligned}
$$

It can be concluded that the Lyapunov matrices $P_{i}$ and $P_{j}$ are coupled with the system matrices. In order to facilitate the controller design, we need to handle with the terms $P_{i}$ and $P_{j}^{-1}$. To this end, for convenience in the controller synthesis, we make a congruence transformation to (26) by $\operatorname{diag}\left\{V_{i}, \mathbf{I}, \mathbf{I}, \mathbf{I}, \mathbf{I}\right\}$ as

$$
\begin{gathered}
{\left[\begin{array}{cccc}
-V_{i} P_{j}^{-1} V_{i}^{\mathrm{T}} & V_{i}\left(\mathrm{a}_{i}+\Delta \mathrm{a}_{i}\right) & V_{i}\left(\mathscr{A}_{i}+\Delta \mathscr{A}_{i}\right) & V_{i} \bar{H}_{i} \\
\star & r_{i j}\left(q_{i}^{\mathrm{T}} q_{i}-1\right) & r_{i j} q_{i}^{\mathrm{T}} \bar{Q}_{i} & \mathbf{0} \\
\star & \star & -P_{i}(\mu)+r_{i j} \bar{Q}_{i}^{\mathrm{T}} \bar{Q}_{i}+\bar{L}_{i}^{\mathrm{T}} \bar{L}_{i} & \mathbf{0} \\
\star & \star & \star & -\gamma^{2} \mathrm{I}
\end{array}\right]} \\
<0, \quad i \in \Pi_{1},(i, j) \in \Omega .
\end{gathered}
$$

Notice that

$$
\begin{aligned}
P_{j} & -V_{i}-V_{i}^{\mathrm{T}}+V_{i} P_{j}^{-1} V_{i}^{\mathrm{T}}=\left(P_{j}-V_{i}\right) P_{j}^{-1}\left(P_{j}-V_{i}\right)^{\mathrm{T}} \\
& \geq 0,
\end{aligned}
$$

which indicates that

$$
-V_{i} P_{j}^{-1} V_{i}^{\mathrm{T}} \leq P_{j}-V_{i}-V_{i}^{\mathrm{T}}
$$

Based on (29), the following inequality implies (27):

$$
\left[\begin{array}{cccc}
P_{j}-V_{i}-V_{i}^{\mathrm{T}} & V_{i}\left(\mathrm{a}_{i}+\Delta \mathrm{a}_{i}\right) & V_{i}\left(\mathscr{A}_{i}+\Delta \mathscr{A}_{i}\right) & V_{i} \bar{H}_{i} \\
\star & r_{i j}\left(q_{i}^{\mathrm{T}} q_{i}-1\right) & r_{i j} q_{i}^{\mathrm{T}} \overline{\mathrm{Q}}_{i} & \mathbf{0} \\
\star & \star & -P_{i}(\mu)+r_{i j} \overline{\mathrm{Q}}_{i}^{\mathrm{T}} \overline{\mathrm{Q}}_{i}+\bar{L}_{i}^{\mathrm{T}} \bar{L}_{i} & \mathbf{0} \\
\star & \star & \star & -\gamma^{2} \mathbf{I}
\end{array}\right]<0, \quad i \in \Pi_{1}, \quad(i, j) \in \Omega .
$$

Notice that the controller gains are not involved in the first row of the matrices $\mathscr{A}_{i}, \Delta \mathscr{A}_{i}, \mathrm{a}_{i}$, and $\Delta \mathrm{a}_{i}$. For numerical tractability, we specify $V_{i}$ as

$$
V_{i}=\left[\begin{array}{cc}
V_{i 1} & \lambda S V_{i 2} \\
V_{i 3} & V_{i 2}
\end{array}\right], \quad i \in \mathscr{I}_{1}
$$

where $\lambda$ and $S$ are defined as in (20).

Based on the matrices defined as in (31), one has that

$$
\begin{aligned}
V_{i} R K_{i} & =\left[\begin{array}{cc}
V_{i 1} & \lambda S V_{i 2} \\
V_{i 3} & V_{i 2}
\end{array}\right]\left[\begin{array}{c}
\mathbf{0} \\
\mathbf{I}_{n_{u}}
\end{array}\right] K_{i}=E \bar{K}_{i}, \\
V_{i} R k_{i} & =\left[\begin{array}{cc}
V_{i 1} & \lambda S V_{i 2} \\
V_{i 3} & V_{i 2}
\end{array}\right]\left[\begin{array}{c}
\mathbf{0} \\
\mathbf{I}_{n_{u}}
\end{array}\right] k_{i}=E \bar{k}_{i},
\end{aligned}
$$

where $R=\left[\begin{array}{ll}\lambda S^{\mathrm{T}} & \mathbf{I}_{n_{u}}\end{array}\right]^{\mathrm{T}}, \bar{K}_{i}=V_{i 2} K_{i}, \bar{k}_{i}=V_{i 2} k_{i}$.
Substitute (32) into (30), and use Lemma 2 to handle the uncertainty terms. Then the following inequality implies (30) for $\varepsilon_{i}>0, i \in \Pi$,

$$
\begin{gathered}
{\left[\begin{array}{cccc}
P_{j}-V_{i}-V_{i}^{\mathrm{T}} & V_{i} \bar{a}_{i}+E \bar{k}_{i} & V_{i} \bar{A}_{s}+R \bar{K}_{i} & V_{i} \bar{H}_{i} \\
\star & r_{i j}\left(q_{i}^{\mathrm{T}} q_{i}-1\right) & r_{i j} q_{i}^{\mathrm{T}} \bar{Q}_{i} & \mathbf{0} \\
\star & \star & -P_{i}+r_{i j} \overline{\mathrm{Q}}_{i}^{\mathrm{T}} \overline{\mathrm{Q}}_{i}+\bar{L}_{i}^{\mathrm{T}} \bar{L}_{i} & \mathbf{0} \\
\star & \star & \star & -\gamma^{2} \mathbf{I}
\end{array}\right]} \\
+\varepsilon_{i}^{-1}\left[\begin{array}{cc}
V_{i} \bar{W}_{i 1} & V_{i} R W_{i 2} \\
\mathbf{0} & \mathbf{0} \\
\mathbf{0} & \mathbf{0} \\
\mathbf{0} & \mathbf{0}
\end{array}\right](\star)+\varepsilon_{i}\left[\begin{array}{cc}
\mathbf{0} & \mathbf{0} \\
M_{i 2}^{\mathrm{T}} & U_{i 2}^{\mathrm{T}} \\
\bar{M}_{i 1}^{\mathrm{T}} & U_{i 1}^{\mathrm{T}} \\
\mathbf{0} & \mathbf{0}
\end{array}\right]<0
\end{gathered}
$$

where

$$
\begin{aligned}
& \bar{W}_{i 1}=\left[\begin{array}{c}
W_{i 1} \\
\mathbf{0}
\end{array}\right], \\
& \bar{M}_{i 1}=\left[\begin{array}{ll}
M_{i 1} & M_{i 3}
\end{array}\right] .
\end{aligned}
$$


On the basis of Schur complement, it is easy to conclude that (19) implies (33). The proof is thus completed.

Remark 4. Note that in most existing nonfragile controller synthesis references for PWA systems, the control input matrices are not empowered to have uncertainties, which is restrictive for the control system design. In addition, it is also difficult to design a nonfragile piecewise affine controller via the approach proposed in [16], as one needs to develop a singular value decomposition to the control input matrices. However, by employing a state-control augmentation strategy for the nonfragile piecewise affine controller design, then the controller gains and the control input matrices can be decoupled from each other. As the state-control augmentation methodology is exploited, the uncertainty terms $\Delta K_{i}$ and $\Delta k_{i}$ in the controller are decoupled from the system matrices. This feature makes it possible to design a piecewise affine nonfragile controller via convexification techniques in a uniform framework.

If the uncertainty terms, affine terms, and external disturbance do not exist, system (1) reduces to a nominal piecewise system as

$$
x(t+1)=A_{i} x(t)+B_{i} u(t) .
$$

For system (35), a piecewise linear nonfragile controller in a differential form is designed as

$$
u(t+1)=\left(K_{i}+\Delta K_{i}\right) \xi(t)
$$

with $\xi(t)$ and $\Delta K_{i}$ defined as in (9).

Substituting the controller (36) into system (35), then the resulting closed-loop system is

$$
\xi(t+1)=\left(\bar{A}_{i}+R K_{i}+R \Delta K_{i}\right) \xi(t)
$$

where $\bar{A}_{i}$ is denoted as in (13).

Corollary 5. For a given constant scalar $\lambda$, the closed-loop system (37) is asymptotically stable if there exist matrices $0<$ $P_{i}=P_{i}^{\mathrm{T}} \in \mathfrak{R}^{(n+m) \times(n+m)}, \bar{K}_{i} \in \mathfrak{R}^{m \times(n+m)}, V_{i 1} \in \mathfrak{R}^{n \times n}$, $V_{i 2} \in \mathfrak{R}^{m \times n}, V_{i 3} \in \mathfrak{R}^{m \times m}$, and $i \in \Pi$ and $a$ set of positive scalar $\varepsilon_{i}>0, i \in \Pi$, such that

$$
\left[\begin{array}{ccc}
P_{j}-V_{i}-V_{\mathrm{i}}^{\mathrm{T}} & V_{i} \bar{A}_{i}+E \bar{K}_{i} & V_{i} R W_{i 2} \\
\star & -P_{i}+\varepsilon_{i} U_{i 1}^{\mathrm{T}} U_{i 1} & 0 \\
\star & \star & -\varepsilon_{i} \mathbf{I}
\end{array}\right]<0,
$$

$$
i \in \Pi \text {. }
$$

Moreover, the controller gain can be obtained by $K_{i}=$ $V_{i 2}^{-1} \bar{K}_{i}$.

The derivation procedures are similar to that of Theorem 3. The proof is omitted.

Remark 6. Note that when the uncertainty terms in the form of (10) in the controller gains do not exist, that is to say,

$$
W_{i 2}=U_{i 1}=U_{i 2}=0, \quad i \in \Pi,
$$

then the piecewise affine nonfragile controller reduces to a regular piecewise affine state-feedback controller as

$$
u(t+1)=K_{i} \xi(t)+k_{i}, \quad i \in \Pi,
$$

where $\xi(t)=\left[\begin{array}{ll}x^{\mathrm{T}}(t) & u^{\mathrm{T}}(t)\end{array}\right]^{\mathrm{T}}$.

\section{Simulation}

In this section, two simulation examples are shown to illustrate the effectiveness of the proposed approaches.

Example 1. Consider a discrete-time piecewise affine system in the form of (1) as follows:

$$
\begin{aligned}
x(t+1)= & \left(A_{i}+\Delta A_{i}\right) x(t)+a_{i}+\Delta a_{i} \\
& +\left(B_{i}+\Delta B_{i}\right) u(t)+H_{i} w(t) \\
z(t)= & L_{i} x(t)+N_{i} u(t), \\
& \quad x(t) \in \Sigma_{i}, i \in\{1,2,3\},
\end{aligned}
$$

and the system matrices are given as

$$
\begin{aligned}
& A_{1}=\left[\begin{array}{cc}
1.2 & 0 \\
-0.1 & 0.4
\end{array}\right] \text {, } \\
& A_{2}=\left[\begin{array}{cc}
0.5 & 0.1 \\
0 & 1.1
\end{array}\right] \text {, } \\
& A_{3}=\left[\begin{array}{ll}
1.1 & 0.3 \\
0.1 & 0.2
\end{array}\right] \text {, } \\
& a_{1}=\left[\begin{array}{c}
0 \\
-0.45
\end{array}\right] \text {, } \\
& a_{2}=\left[\begin{array}{l}
0 \\
0
\end{array}\right] \text {, } \\
& a_{3}=\left[\begin{array}{c}
0 \\
0.45
\end{array}\right] \text {, } \\
& B_{1}=\left[\begin{array}{c}
1 \\
0.5
\end{array}\right] \text {, } \\
& B_{2}=\left[\begin{array}{c}
1 \\
1.5
\end{array}\right] \text {, } \\
& B_{3}=\left[\begin{array}{l}
0.9 \\
1.2
\end{array}\right] \text {, } \\
& H_{1}=\left[\begin{array}{l}
0.8 \\
0.5
\end{array}\right] \text {, }
\end{aligned}
$$




$$
\begin{aligned}
& H_{2}=\left[\begin{array}{l}
0.7 \\
0.6
\end{array}\right] \text {, } \\
& H_{3}=\left[\begin{array}{l}
0.4 \\
0.4
\end{array}\right] \text {, } \\
& L_{1}=\left[\begin{array}{ll}
1 & 0.3
\end{array}\right] \text {, } \\
& L_{2}=\left[\begin{array}{ll}
1.2 & 0.5
\end{array}\right] \text {, } \\
& L_{3}=\left[\begin{array}{ll}
0.5 & 1
\end{array}\right] \text {, } \\
& N_{1}=1 \text {, } \\
& N_{2}=0.8 \text {, } \\
& N_{3}=1.2 \text {, } \\
& W_{11}=\left[\begin{array}{l}
0.1 \\
0.2
\end{array}\right] \text {, } \\
& W_{21}=\left[\begin{array}{l}
0.3 \\
0.1
\end{array}\right] \text {, } \\
& W_{31}=\left[\begin{array}{l}
0.4 \\
0.1
\end{array}\right] \text {, } \\
& M_{11}=\left[\begin{array}{ll}
0.01 & 0.01
\end{array}\right] \text {, } \\
& M_{21}=\left[\begin{array}{ll}
0.01 & 0.03
\end{array}\right] \text {, } \\
& M_{31}=\left[\begin{array}{ll}
0.03 & 0.02
\end{array}\right] \text {, } \\
& M_{12}=0.1 \text {, } \\
& M_{22}=0.2 \text {, } \\
& M_{32}=0.05 \text {, } \\
& M_{i 3}=0.01 \text {, }
\end{aligned}
$$

and the parameter uncertainties in controllers in terms of (10) are

$$
\begin{aligned}
W_{i 2} & =0.8, \\
U_{i 1} & =\left[\begin{array}{lll}
0.8 & 0.8 & 0.8
\end{array}\right], \\
U_{i 2} & =0.8,
\end{aligned}
$$

$$
i \in\{1,2,3\} \text {. }
$$

The decomposed regions are given as

$$
\begin{aligned}
& \Sigma_{1}=\left\{x(t) \mid-\beta<x_{1}(t) \leq-\alpha\right\}, \\
& \Sigma_{2}=\left\{x(t) \mid-\alpha<x_{1}(t) \leq \alpha\right\}, \\
& \Sigma_{3}=\left\{x(t) \mid \alpha<x_{1}(t) \leq \beta\right\},
\end{aligned}
$$

where $\alpha=40$ and $\beta=400$.
From the relationship in (7), we can obtain that $\phi_{i}^{\mathrm{T}}=$ $\left[\begin{array}{ll}1 & 0\end{array}\right]$, for $i \in \Pi$, and the parameters of the degenerate ellipsoids are

$$
\begin{aligned}
Q_{1} & =\frac{2 \phi_{i}^{\mathrm{T}}}{\beta-\alpha}, \\
q_{1} & =\frac{\beta+\alpha}{\beta-\alpha}, \\
Q_{2} & =\frac{1}{d_{1}}, \\
q_{2} & =0, \\
Q_{3} & =\frac{2 \phi_{i}^{\mathrm{T}}}{\beta-\alpha}, \\
q_{3} & =\frac{\beta+\alpha}{\alpha-\beta} .
\end{aligned}
$$

The objective is to design a piecewise affine nonfragile controller in the form of (9)-(10) such that the closed-loop system in (12) is asymptotically stable with a robust $\mathscr{H}_{\infty}$ performance $\gamma$. Exploiting Theorem 3 with $S=\left[\begin{array}{ll}1 & 0\end{array}\right]^{\mathrm{T}}$ and $\lambda=1$, the controller gains can be obtained as

$$
\begin{aligned}
K_{1} & =\left[\begin{array}{lll}
-1.3002 & -0.1249 & -1.2656
\end{array}\right], \\
k_{1} & =0.2580, \\
K_{2} & =\left[\begin{array}{lll}
-1.5930 & -0.1369 & -1.5294
\end{array}\right], \\
k_{2} & =0, \\
K_{3} & =\left[\begin{array}{lll}
-1.5756 & -0.1317 & -1.5052
\end{array}\right], \\
k_{3} & =-0.1308,
\end{aligned}
$$

with robust $\mathscr{H}_{\infty}$ performance $\gamma_{\min }=1.4222$.

It is worth pointing out that when $k_{i} \equiv 0$, the piecewise affine nonfragile controller (9) is characterized as a piecewise linear nonfragile controller given in (36). With $\lambda=1$, Table 1 compares the robust performance between piecewise affine nonfragile controllers with piecewise linear nonfragile controllers with different matrix $S$.

It can be seen from Table 1 that the piecewise affine nonfragile controllers have a better robust $\mathscr{H}_{\infty}$ performance than the piecewise linear nonfragile controllers.

To verify the effectiveness of the designed controllers, simulations are carried out. With $S=\left[\begin{array}{ll}1 & 0\end{array}\right]^{\mathrm{T}}$ and $\lambda=1$, solving the LMIs in (18)-(19), we obtain the piecewise linear nonfragile controller gains as

$$
\begin{aligned}
& K_{1}=\left[\begin{array}{lll}
-1.3003 & -0.1261 & -1.2658
\end{array}\right], \\
& K_{2}=\left[\begin{array}{lll}
-1.5903 & -0.1367 & -1.5266
\end{array}\right], \\
& K_{3}=\left[\begin{array}{lll}
-1.5736 & -0.1302 & -1.5033
\end{array}\right] .
\end{aligned}
$$

In this simulation example, the initial condition is $x_{0}=$ $\left[\begin{array}{ll}2 & -1.5\end{array}\right]^{\mathrm{T}}$, and the external disturbance is $w(t)=120 \pi^{-3.6 t}$. 
TABLE 1: Comparison of robust $\mathscr{H}_{\infty}$ performance for piecewise-affine/piecewise-linear nonfragile controllers.

\begin{tabular}{lccc}
\hline Controller form & $S=\left[\begin{array}{ll}1 & 0\end{array}\right]^{\mathrm{T}}$ & $S=\left[\begin{array}{ll}1 & 1\end{array}\right]^{\mathrm{T}}$ & $S=\left[\begin{array}{ll}1 & -1\end{array}\right]^{\mathrm{T}}$ \\
\hline piecewise affine controller & 1.4222 & 1.6797 & 4.6871 \\
piecewise linear controller & 1.5924 & 1.7997 & 4.7362 \\
\hline
\end{tabular}

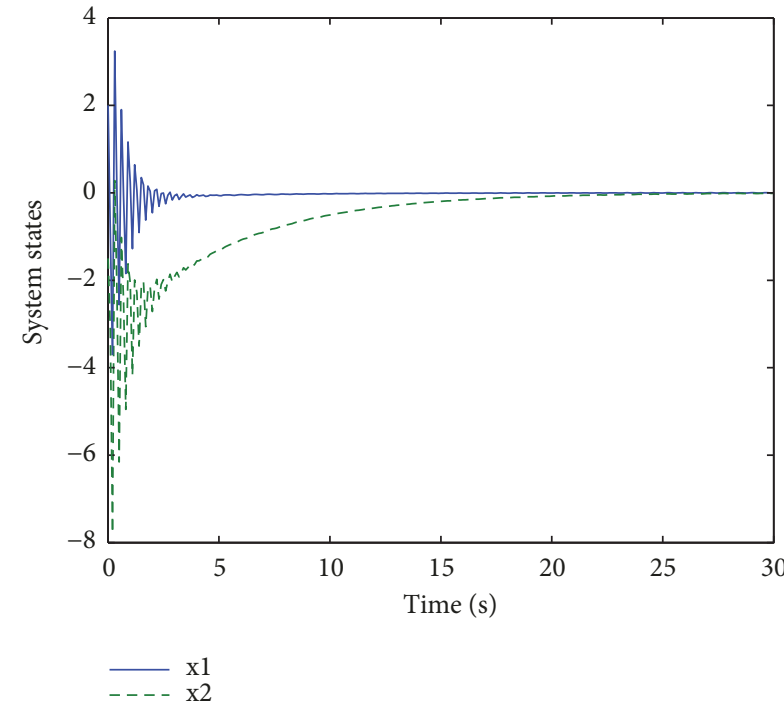

(a) State response

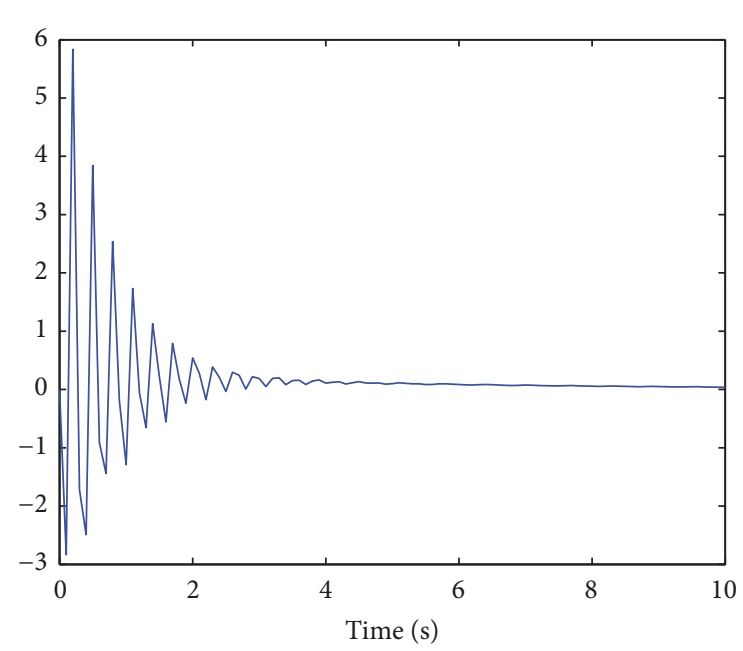

$\mathrm{u}(\mathrm{t})$

FIGURE 1: Simulation results in Example 1 by using piecewise affine nonfragile controller (9).

$\cos (4 t)$. By using the piecewise affine nonfragile controller in (9), the state response of the closed-loop system is demonstrated in Figure 1(a), and the control input is shown in Figure 1(b). It can be seen from Figure 1(a) that the curves of system states converge to the zero after 20 s.

With the same initial conditions and external disturbance, use the piecewise linear nonfragile controller as in (36), and the time responses of the closed-loop system are demonstrated in Figures 2(a) and 2(b). Figure 2(a) shows that the curves of the closed-loop system states converge to the zero after $28 \mathrm{~s}$.

Compare Figure 1(a) with Figure 2(a), and one can conclude that the convergence rate of the closed-loop system states with using piecewise affine nonfragile controller is faster than those with using piecewise linear nonfragile controller.

Under zero initial conditions, Figure 3 presents the $\mathscr{H}_{\infty}$ performance. The ratio $\sqrt{\int_{0}^{t_{f}} z^{\mathrm{T}}(t) z(t) d t} / \sqrt{\int_{0}^{t_{f}} w^{\mathrm{T}}(t) w(t) d t}$ with using piecewise affine nonfragile controller is about 1.18, which is lower than $\gamma_{\min }=1.4222$. The ratio $\sqrt{\int_{0}^{t_{f}} z^{\mathrm{T}}(t) z(t) d t} / \sqrt{\int_{0}^{t_{f}} w^{\mathrm{T}}(t) w(t) d t}$ with using piecewise linear nonfragile controller is about 1.3, which is lower than $\gamma_{\min }=1.5924$. It can also be seen that a better disturbance attenuation level can be obtained with the piecewise affine nonfragile controller.

In order to verify the advantages of piecewise affine nonfragile controller (9) over the piecewise affine statefeedback controller (40), another simulation is conducted.
Solve the LMIs in (18)-(19) with $W_{i 2}=0, U_{i 1}=\left[\begin{array}{lll}0 & 0 & 0\end{array}\right]$, and $U_{i 2}=0, i \in\{1,2,3\}$. Feasible solutions can be obtained with $\lambda=1$ and $S=\left[\begin{array}{ll}1 & 0\end{array}\right]^{\mathrm{T}}$, and the controller gains are

$$
\begin{aligned}
K_{1} & =\left[\begin{array}{lll}
-1.3202 & -0.1148 & -1.2689
\end{array}\right], \\
k_{1} & =0.2038, \\
K_{2} & =\left[\begin{array}{lll}
-1.4634 & -0.1183 & -1.3938
\end{array}\right], \\
k_{2} & =0, \\
K_{3} & =\left[\begin{array}{lll}
-1.4888 & -0.1168 & -1.4112
\end{array}\right], \\
k_{3} & =-0.1174 .
\end{aligned}
$$

With the same initial conditions, disturbance, and parameter variations in the controller gains, apply controller (40) to system (41), and the trajectories of the closed-loop system states are shown in Figure 4. It can be seen from Figure 4 that the piecewise affine state-feedback controller (40) can not stabilize system (39) with parameter variations existing in the controller gains. From this case, we can see that the regular state-feedback controller can not deal with the situations that parameter variations exist in the controller gains.

This example clearly illustrates the advantages of the proposed piecewise affine nonfragile controller over the piecewise linear nonfragile controller and the piecewise affine state-feedback controller. 


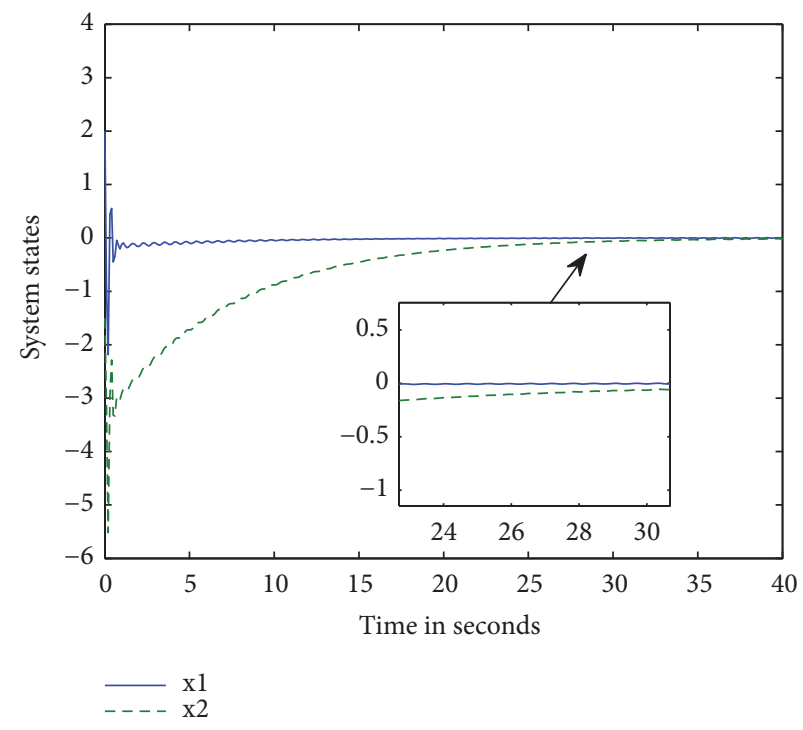

(a) State response

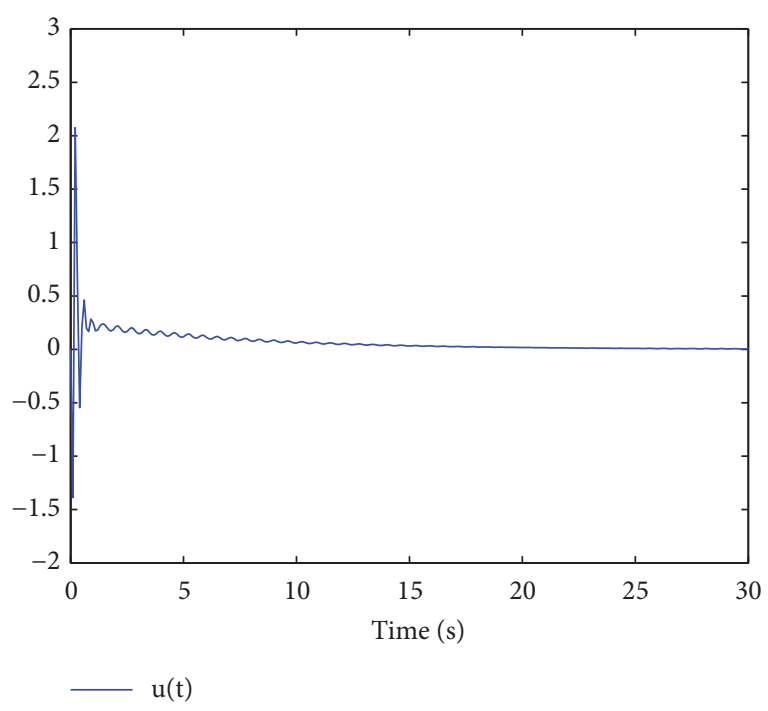

(b) Control input

FIGURE 2: Simulation results in Example 1 by using piecewise linear nonfragile controller (36).

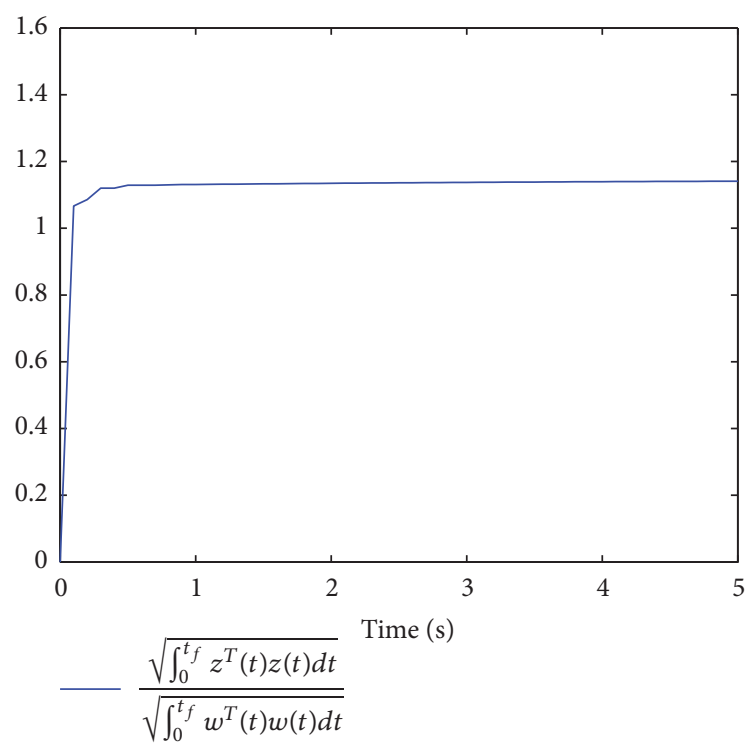

(a) Response of the ratio by using piecewise affine nonfragile controller (9)

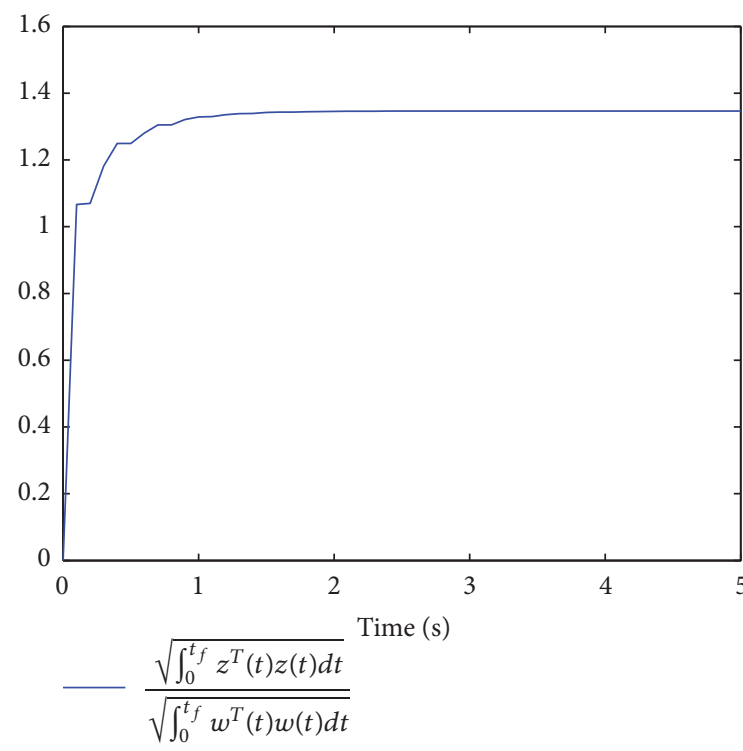

(b) Response of the ratio by using piecewise affine nonfragile controller (36)

Figure 3: Response of the ratio $\sqrt{\int_{0}^{t_{f}} z^{\mathrm{T}}(t) z(t) d t} / \sqrt{\int_{0}^{t_{f}} w^{\mathrm{T}}(t) w(t) d t}$ in Example 1.

Example 2. Consider another discrete-time Chuas circuit system represented by the piecewise affine model as

$$
\begin{aligned}
x(t+1) & =A_{i} x(t)+a_{i}+B_{i} u(t)+H_{i} w(t) \\
z(t) & =L_{i} x(t)+N_{i} u(t), \quad x(t) \in \Sigma_{i}, i \in\{1,2,3\}
\end{aligned}
$$

where the system matrices are

$$
A_{1}=\left[\begin{array}{ccc}
0.9955 & 0.0070 & 0 \\
0.0007 & 0.9993 & -0.0011 \\
0 & 0.0074 & 1
\end{array}\right],
$$

$$
\begin{aligned}
& A_{2}=\left[\begin{array}{ccc}
0.9983 & 0.0070 & 0 \\
0.0007 & 0.9993 & -0.0011 \\
0 & 0.0074 & 1
\end{array}\right], \\
& A_{3}=\left[\begin{array}{ccc}
0.9955 & 0.0070 & 0 \\
0.0007 & 0.9993 & -0.0011 \\
0 & 0.0074 & 1
\end{array}\right], \\
& a_{1}=\left[\begin{array}{c}
0.0028 \\
0 \\
0
\end{array}\right],
\end{aligned}
$$




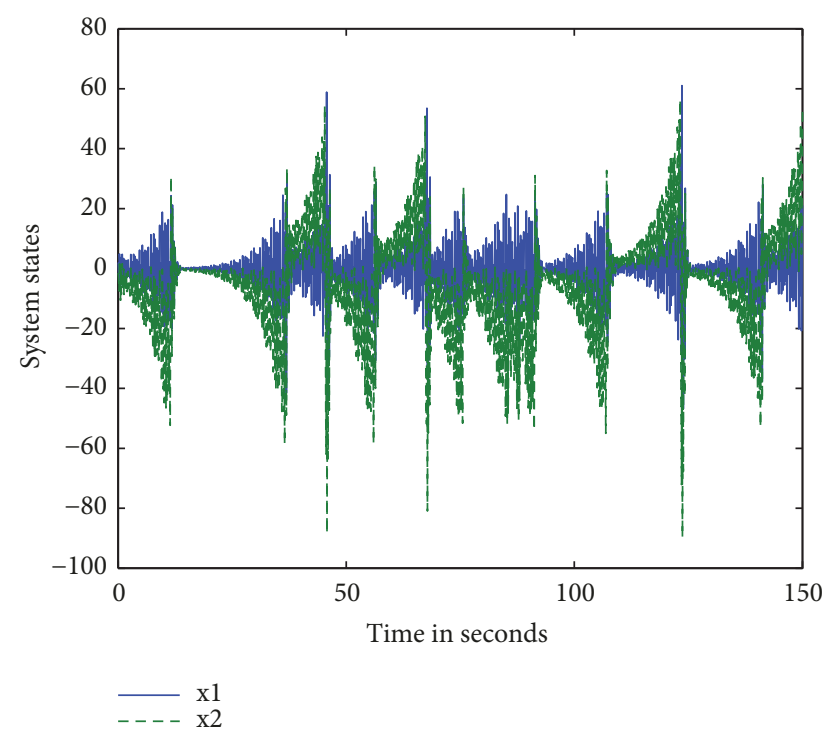

FIgURE 4: State responses in Example 1 by using piecewise affine state-feedback controller (40).

$$
\begin{aligned}
& a_{2}=\left[\begin{array}{l}
0 \\
0 \\
0
\end{array}\right], \\
& a_{3}=\left[\begin{array}{c}
-0.0028 \\
0 \\
0
\end{array}\right] \text {, } \\
& B_{1}=B_{3}\left[\begin{array}{c}
-0.00998 \\
0 \\
0
\end{array}\right] \text {, } \\
& B_{2}=\left[\begin{array}{c}
-0.00999 \\
0 \\
0
\end{array}\right] \text {, } \\
& H_{1}=H_{3}=\left[\begin{array}{l}
0 \\
2 \\
0
\end{array}\right] \text {, } \\
& H_{2}=\left[\begin{array}{c}
0.5 \\
0 \\
0
\end{array}\right] \text {, } \\
& L_{1}=\left[\begin{array}{lll}
1 & 0 & 0
\end{array}\right] \text {, } \\
& L_{2}=\left[\begin{array}{lll}
0.5 & 0 & 0
\end{array}\right] \text {, } \\
& L_{3}=\left[\begin{array}{lll}
1.5 & 0 & 0
\end{array}\right] \text {, } \\
& N_{i}=0, \quad i \in\{1,2,3\} \text {. }
\end{aligned}
$$

The decomposed regions are given as

$$
\begin{aligned}
& \Sigma_{1}=\left\{x(t) \mid-\beta<x_{1}(t) \leq-\alpha\right\}, \\
& \Sigma_{2}=\left\{x(t) \mid-\alpha<x_{1}(t) \leq \alpha\right\}, \\
& \Sigma_{3}=\left\{x(t) \mid \alpha<x_{1}(t) \leq \beta\right\},
\end{aligned}
$$

where $\alpha=1, \beta=5$.

From (7), we can obtain that $\phi_{i}^{\mathrm{T}}=\left[\begin{array}{lll}1 & 0 & 0\end{array}\right]$, for $i \in \Pi$, and the parameters of the degenerate ellipsoid are

$$
\begin{aligned}
Q_{1} & =\left[\begin{array}{lll}
0.5 & 0 & 0
\end{array}\right], \\
q_{1} & =-1.5, \\
Q_{2} & =\left[\begin{array}{lll}
1 & 0 & 0
\end{array}\right], \\
q_{2} & =0, \\
Q_{3} & =\left[\begin{array}{lll}
0.5 & 0 & 0
\end{array}\right], \\
q_{3} & =1.5 .
\end{aligned}
$$

It is assumed in this example that the perturbations existing in the controller gains are set as

$$
\begin{aligned}
M_{i 2} & =0.1, \\
U_{i 1} & =\left[\begin{array}{llll}
0.1 & 0.1 & 0.1 & 0.1
\end{array}\right], \\
U_{i 2} & =0.3,
\end{aligned}
$$

$$
i \in\{1,2,3\} \text {. }
$$

As the controller gains contain parameter perturbations, we will design a piecewise affine nonfragile controller in (9) to guarantee that the closed-loop system is asymptotically stabile with a robust $\mathscr{H}_{\infty}$ performance $\gamma$. Exploiting Theorem 3 with $S=\left[\begin{array}{ll}1 & 0\end{array}\right]^{\mathrm{T}}$ and $\lambda=1$, the controller gains can be obtained as

$$
\begin{aligned}
& K_{1}=\left[\begin{array}{llll}
-0.1688 & -0.0053 & -0.1464 & 0.0017
\end{array}\right], \\
& k_{1}=-4.7949 \times 10^{-4} \text {, } \\
& K_{2}=\left[\begin{array}{llll}
-0.0147 & -0.0005 & -0.0127 & 0.0001
\end{array}\right] \text {, } \\
& k_{2}=0 \text {, } \\
& K_{3}=\left[\begin{array}{llll}
-0.0204 & -0.0006 & -0.0176 & 0.0002
\end{array}\right] \text {, } \\
& k_{3}=5.7296 \times 10^{-5} \text {, }
\end{aligned}
$$

with robust $\mathscr{H}_{\infty}$ performance $\gamma_{\min }=0.2755$.

To present the effectiveness of the designed controllers, simulations are carried out. With initial conditions $x_{0}=$ $\left[\begin{array}{lll}3.5 & 0 & -3\end{array}\right]^{\mathrm{T}}$ and the external disturbance $w(t)=120 \pi^{-3.6 t}$. $\cos (4 t)$, the state trajectories of the closed-loop system are demonstrated in Figure 5(a).

Solving the LMIs in (18)-(19) with $W_{i 2}=0, U_{i 1}=$ $\left[\begin{array}{lll}0 & 0 & 0\end{array}\right], U_{i 2}=0, i \in\{1,2,3\}$, and $\lambda=1, S=\left[\begin{array}{lll}2 & 2 & 1\end{array}\right]^{\mathrm{T}}$, then 


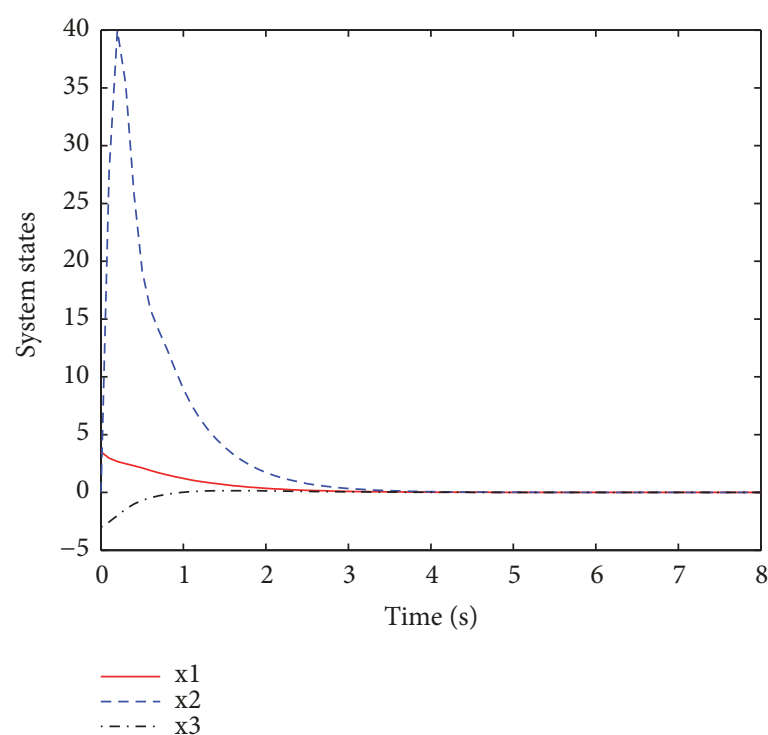

(a) Response of closed-loop system states by using piecewise affine nonfragile controller (9)

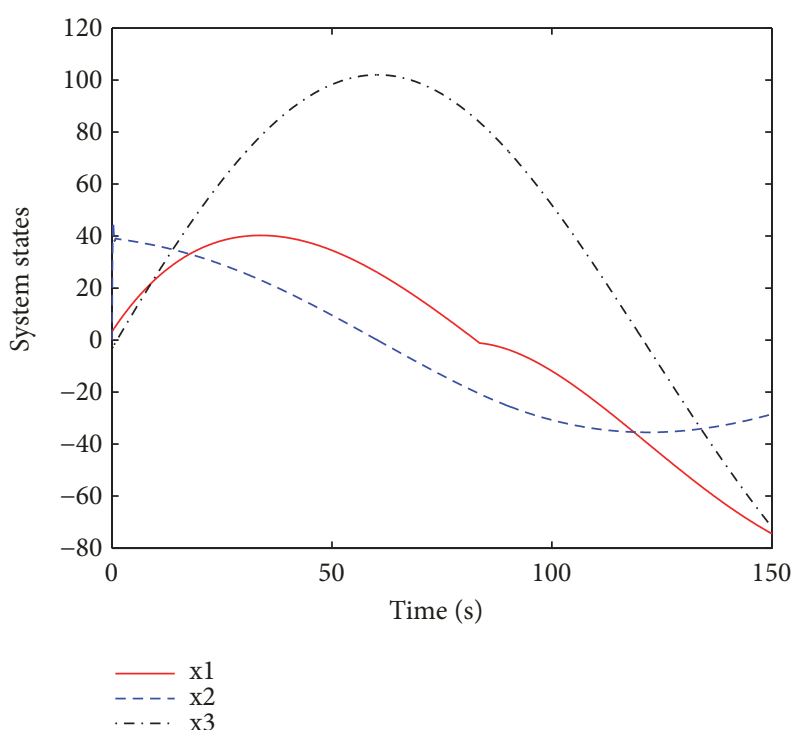

(b) Response of closed-loop system states by using piecewise affine nonfragile controller (40)

FIgURE 5: Response of the system states in Example 2.

the gains of the piecewise affine state-feedback controller (40) are

$$
\begin{aligned}
& K_{1}=\left[\begin{array}{llll}
-0.1407 & -0.0041 & -0.1471 & 0.0014
\end{array}\right], \\
& k_{1}=1.3805 \times 10^{-6} \text {, } \\
& K_{2}=\left[\begin{array}{llll}
-0.1688 & -0.0050 & -0.1772 & 0.0017
\end{array}\right] \text {, } \\
& k_{2}=0 \text {, } \\
& K_{3}=\left[\begin{array}{llll}
-0.1536 & -0.0045 & -0.1613 & 0.0015
\end{array}\right] \times 10^{-6}, \\
& k_{3}=4.3196 \times 10^{-10} \text {. }
\end{aligned}
$$

With the same initial conditions and disturbance, we use controller (40) to system (49), and the closed-loop system states are demonstrated in Figure 5(b). It can be seen from Figure 5 that the piecewise affine state-feedback controller (40) can not stabilize system (49) with parameter variations existing in the controller gains.

\section{Conclusion}

In this paper, we have studied the nonfragile control problem for a class of discrete-time hybrid systems based on piecewise affine model. Based on PLFs, some new sufficient conditions for piecewise affine nonfragile controller synthesis are presented. By virtue of several convexification strategies, the controller gains can be attained via solving a set of linear matrix inequalities. Finally, simulation examples are carried out to demonstrate the advantages of the proposed methodologies.

\section{Data Availability}

The data used to support the findings of this study are available from the corresponding author upon request.

\section{Conflicts of Interest}

The authors declare that there are no conflicts of interest regarding the publication of this paper.

\section{Acknowledgments}

This work is financially supported by the National Key Research and Development Program (Grants nos. 2017YFC0306804 and 2017YFC0305700) and State Key Laboratory of Ocean Engineering (Shanghai Jiao Tong University) (Grant no. 1715).

\section{References}

[1] A. Bemporad, G. Ferrari-Trecate, and M. Morari, "Observability and controllability of piecewise affine and hybrid systems," IEEE Transactions on Automatic Control, vol. 45, no. 10, pp. 18641876, 2000.

[2] A. Bemporad and M. Morari, "Control of systems integrating logic, dynamics, and constraints," Automatica, vol. 35, no. 3, pp. 407-427, 1999.

[3] A. N. Vargas, L. P. Sampaio, L. Acho, L. Zhang, and J. B. R. Do Val, "Optimal control of DC-DC buck converter via linear systems with inaccessible markovian jumping modes," IEEE Transactions on Control Systems Technology, vol. 24, no. 5, pp. 1820-1827, 2016.

[4] L. Acho, J. Rodellar, C. Tutivi, and Y. Vidal, "Passive fault tolerant control strategy in controlled wind turbines," in Proceeding 
of the 3rd Conference on Control and Fault-Tolerant Systems (SysTol), pp. 636-641, Barcelona, Spain, 2016.

[5] Y. L. Wei, M. Wang, and J. B. Qiu, "New approach to delaydependent $H_{\infty}$ filtering for discrete-time Markovian jump systems with time-varying delay and incomplete transition descriptions," IET Control Theory \& Applications, vol. 7, no. 5, pp. 684-696, 2013.

[6] Y. Wei, J. H. Park, H. R. Karimi, Y.-C. Tian, and H. Jung, "Improved stability and stabilization results for stochastic synchronization of continuous-time semi-markovian jump neural networks with time-varying delay," IEEE Transactions on Neural Networks and Learning Systems, vol. 29, no. 6, pp. 2488-2501, 2017.

[7] D. Mignone, G. Ferrari-Trecate, and M. Morari, "Stability and stabilization of piecewise affine and hybrid systems: An LMI approach," in Proceedings of the 39th IEEE Confernce on Decision and Control, pp. 504-509, Australia, December 2000.

[8] X. Zhao, "Piecewise Ho static output feedback controller design for nonlinear systems based on T-S affine fuzzy models," Mathematical Problems in Engineering, vol. 2015, Article ID 734934, 9 pages, 2015.

[9] Z. Zhou, M. Wang, and Q. Yin, "Robust H-infinity stabilization and resilient filtering for discrete-time constrained singular piecewise-affine systems," Mathematical Problems in Engineering, vol. 2015, Article ID 878120, 16 pages, 2015.

[10] M. Johansson and A. Rantzer, "Computation of piecewise quadratic Lyapunov functions for hybrid systems," IEEE Transactions on Automatic Control, vol. 43, no. 4, pp. 555-559, 1998.

[11] J. Qiu, Y. Wei, and L. Wu, "A novel approach to reliable control of piecewise affine systems with actuator faults," IEEE Transactions on Circuits and Systems II: Express Briefs, vol. 64, no. 8, pp. 957961, 2017.

[12] S. Pettersson and B. Lennartson, "Exponential stability of hybrid systems using piecewise quadratic Lyapunov functions resulting in LMIS," IFAC Proceedings Volumes, vol. 32, no. 2, pp. 48104815, 1999.

[13] J. Imura, "Classification and stabilizability analysis of bimodal piecewise affine systems," International Journal of Robust and Nonlinear Control, vol. 12, no. 10, pp. 897-926, 2002.

[14] G. Ferrari-Trecate, F. A. Cuzzola, D. Mignone, and M. Morari, "Analysis of discrete-time piecewise affine and hybrid systems," Automatica, vol. 38, no. 12, pp. 2139-2146, 2002.

[15] Z. Tang, J. H. Park, Y. Wang, and J. Feng, "Distributed impulsive quasi-synchronization of Lure networks with proportional delay," IEEE Transactions on Cybernetics, 2018.

[16] X. Yao, J. H. Park, H. Dong, L. Guo, and X. Lin, "Robust adaptive nonsingular terminal sliding mode control for automatic train operation," IEEE Transactions on Systems, Man, and Cybernetics: Systems, pp. 1-10, 2017.

[17] X. Xie, N. Duan, and C. Zhao, "A combined homogeneous domination and sign function approach to output-feedback stabilization of stochastic high-order nonlinear systems," IEEE Transactions on Automatic Control, vol. 59, no. 5, pp. 1303-1309, 2014.

[18] L. Xue, T. Zhang, W. Zhang, and X. Xie, "Global adaptive stabilization and tracking control for high-order stochastic nonlinear systems with time-varying delays," IEEE Transactions on Automatic Control, vol. 63, no. 9, pp. 2928-2943, 2018.

[19] A. Hamadeh and J. Goncalves, "Reachability analysis of continuous-time piecewise affine systems," Automatica, vol. 44, no. 12, pp. 3189-3194, 2008.
[20] J. Qiu, G. Feng, and H. Gao, "Approaches to robust $H_{\infty}$ static output feedback control of discrete-time piecewise-affine systems with norm-bounded uncertainties," International Journal of Robust and Nonlinear Control, vol. 21, no. 7, pp. 790-814, 2011.

[21] J. H. Richter, W. P. M. H. Heemels, N. van de Wouw, and J. Lunze, "Reconfigurable control of piecewise affine systems with actuator and sensor faults: stability and tracking," Automatica, vol. 47, no. 4, pp. 678-691, 2011.

[22] Y. Wei, J. Qiu, P. Shi, and H.-K. Lam, “A New Design of $h$-Infinity Piecewise Filtering for Discrete-Time Nonlinear Time-Varying Delay Systems via T-S Fuzzy Affine Models," IEEE Transactions on Systems, Man, and Cybernetics: Systems, vol. 47, no. 8, pp. 2034-2047, 2017.

[23] Y. Wei, J. Qiu, P. Shi, and M. Chadli, "Fixed-order piecewiseaffine output feedback controller for fuzzy-affine-model-based nonlinear systems with time-varying delay," IEEE Transactions on Circuits and Systems I: Regular Papers, vol. 64, no. 4, pp. 945958, 2017.

[24] Y. Wei, J. Qiu, and H.-K. Lam, "A novel approach to reliable output feedback control of fuzzy-affine systems with time delays and sensor faults," IEEE Transactions on Fuzzy Systems, vol. 25, no. 6, pp. 1808-1823, 2017.

[25] J. C. Doyle, K. Glover, P. P. Khargonekar, and B. A. Francis, "State-space solutions to standard $\mathrm{H}_{2}$ and $\mathrm{H} \infty \mathrm{o}$ control problems," IEEE Transactions on Automatic Control, vol. 34, no. 8, pp. 831-847, 1989.

[26] S. Liu, C. Xu, and L. Zhang, "Robust course keeping control of a fully submerged hydrofoil vessel without velocity measurement: an iterative learning approach," Mathematical Problems in Engineering, vol. 2017, Article ID 7979438, 14 pages, 2017.

[27] L. H. Keel and S. P. Bhattacharyya, "Robust, fragile, or optimal?" IEEE Transactions on Automatic Control, vol. 42, no. 8, pp. 10981105, 1997.

[28] P. Dorato, "Non-fragile controller design: an overview," in Proceedings of the American Control Conference (ACC '98), vol. 5, pp. 2829-2831, June 1998.

[29] Y.-Q. Wu, H. Y. Su, R. Q. Lu, Z.-G. Wu, and Z. Shu, "Passivitybased non-fragile control for Markovian jump systems with aperiodic sampling," Systems \& Control Letters, vol. 84, pp. 3543, 2015.

[30] H. Shen, F. Li, Z. Wu, J. H. Park, and V. Sreeram, "Fuzzy-modelbased non-fragile control for nonlinear singularly perturbed systems with semi-markov jump parameters," IEEE Transactions on Fuzzy Systems, pp. 1-1, 2018.

[31] G.-H. Yang and J. L. Wang, "Non-fragile $H_{\infty}$ control for linear systems with multiplicative controller gain variations," Automatica, vol. 37, no. 5, pp. 727-737, 2001.

[32] J. H. Park, "Robust non-fragile control for uncertain discretedelay large-scale systems with a class of controller gain variations," Applied Mathematics and Computation, vol. 149, no. 1, pp. 147-164, 2004.

[33] B. Zhang, S. Zhou, and T. Li, "A new approach to robust and non-fragile $H_{\infty}$ control for uncertain fuzzy systems," Information Sciences, vol. 177, no. 22, pp. 5118-5133, 2007.

[34] J. Yang, S. Zhong, and L. Xiong, "A descriptor system approach to non-fragile $H_{\infty}$ control for uncertain fuzzy neutral systems," Fuzzy Sets and Systems, vol. 160, no. 4, pp. 423-438, 2009.

[35] L. Li and Y. Jia, "Non-fragile dynamic output feedback control for linear systems with time-varying delay," IET Control Theory \& Applications, vol. 3, no. 8, pp. 995-1005, 2009. 
[36] R. Rakkiyappan, R. Sasirekha, S. Lakshmanan, and C. P. Lim, "Synchronization of discrete-time Markovian jump complex dynamical networks with random delays via non-fragile control," Journal of The Franklin Institute, vol. 353, no. 16, pp. 43004329, 2016.

[37] N. Sakthivel, R. Rakkiyappan, and J. H. Park, "Non-fragile synchronization control for complex networks with additive time-varying delays," Complexity, vol. 21, no. 1, pp. 296-321, 2015.

[38] Y. Yu, H. Dong, Z. Wang, W. Ren, and F. E. Alsaadic, "Design of non-fragile state estimators for discrete time-delayed neural networks with parameter uncertainties," Neurocomputing, vol. 182, pp. 18-24, 2016.

[39] S. He, "Non-fragile passive controller design for nonlinear Markovian jumping systems via observer-based controls," $\mathrm{Neu}$ rocomputing, vol. 147, pp. 350-357, 2015.

[40] M. Kchaou, A. E. Hajjaji, and A. Toumi, "Non-fragile Ho output feedback control design for continuous-time fuzzy systems," ISA Transactions ${ }^{\circledR}$, vol. 54, pp. 3-14, 2015.

[41] N. Dadkhah and L. Rodrigues, "Non-fragile state-feedback control of uncertain piecewise-affine slab systems with input constraints: a convex optimisation approach," IET Control Theory \& Applications, vol. 8, no. 8, pp. 626-632, 2014.

[42] W. Ji, A. Wang, and J. Qiu, "A novel dynamic state feedback controller for discrete-time T-S fuzzy affine systems," in Proceedings of the 2017 Seventh International Conference on Information Science and Technology (ICIST), pp. 1-7, Da Nang, Vietnam, April 2017. 


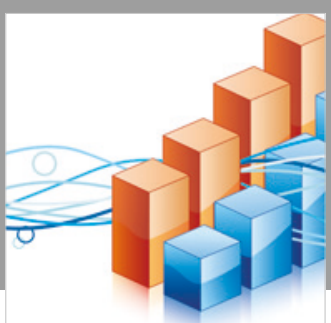

Advances in

Operations Research

\section{-n-m}
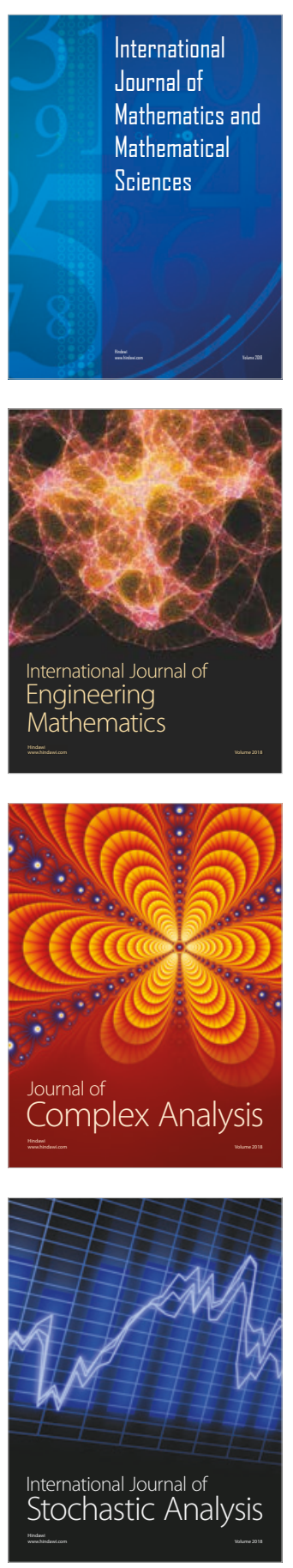
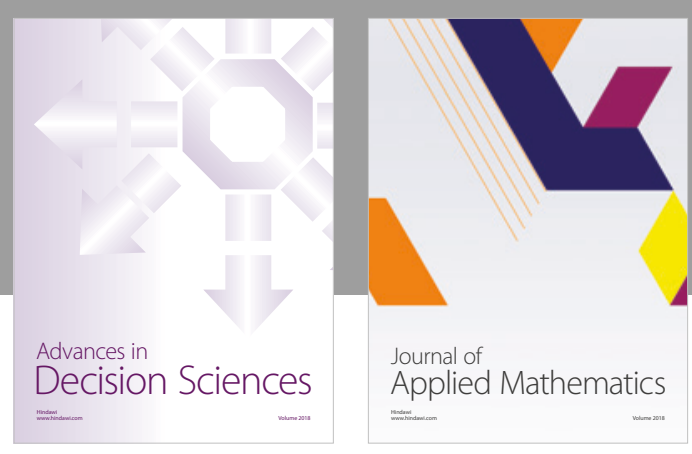

Journal of

Applied Mathematics
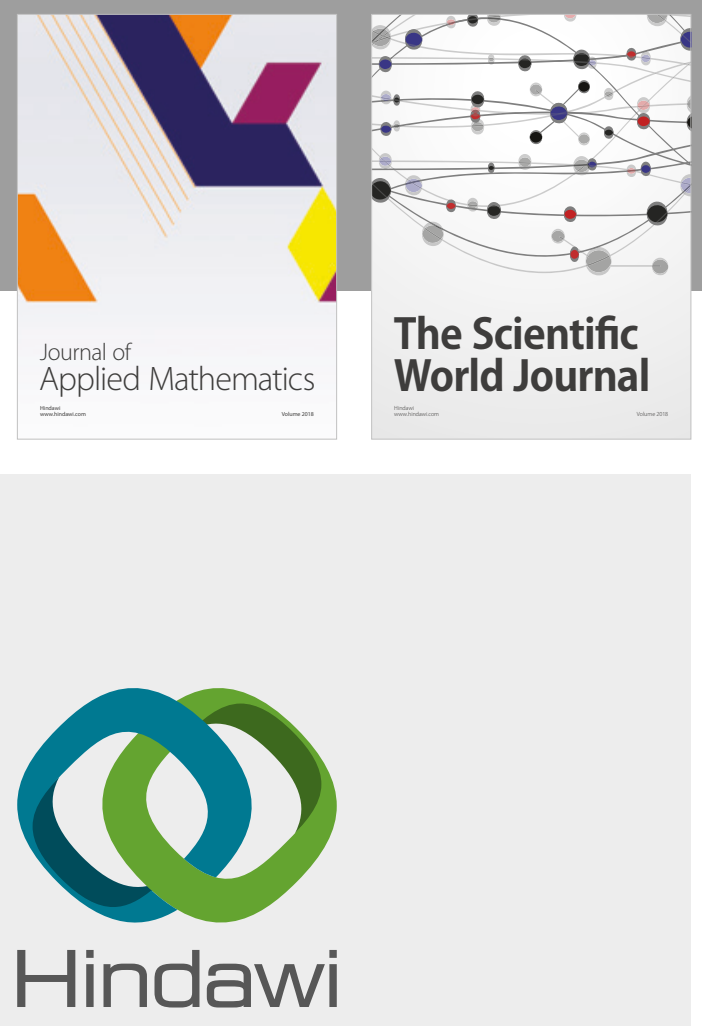

Submit your manuscripts at

www.hindawi.com

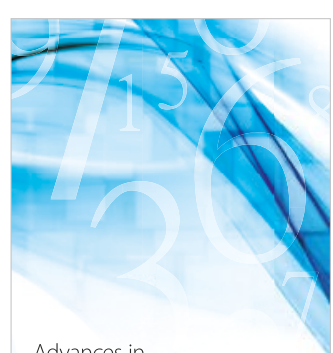

Advances in
Numerical Analysis
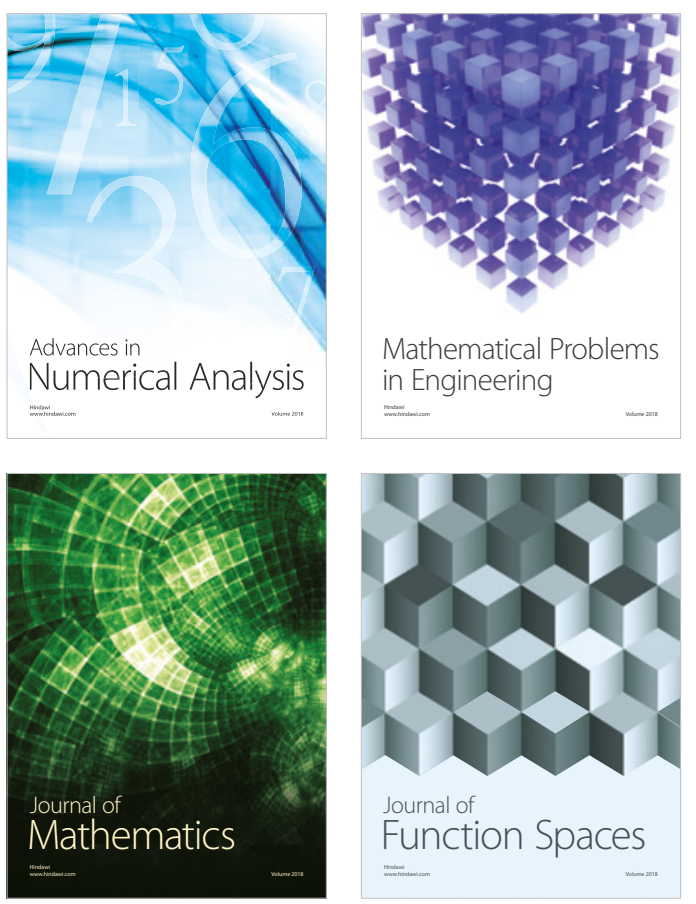

Mathematical Problems in Engineering

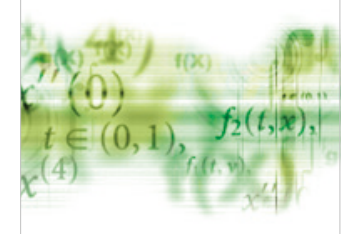

International Journal of

Differential Equations

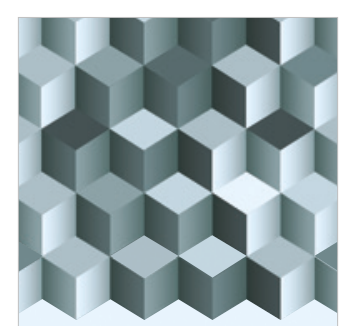

Journal of

Function Spaces
The Scientific

World Journal

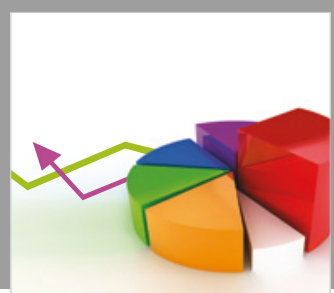

Journal of

Probability and Statistics
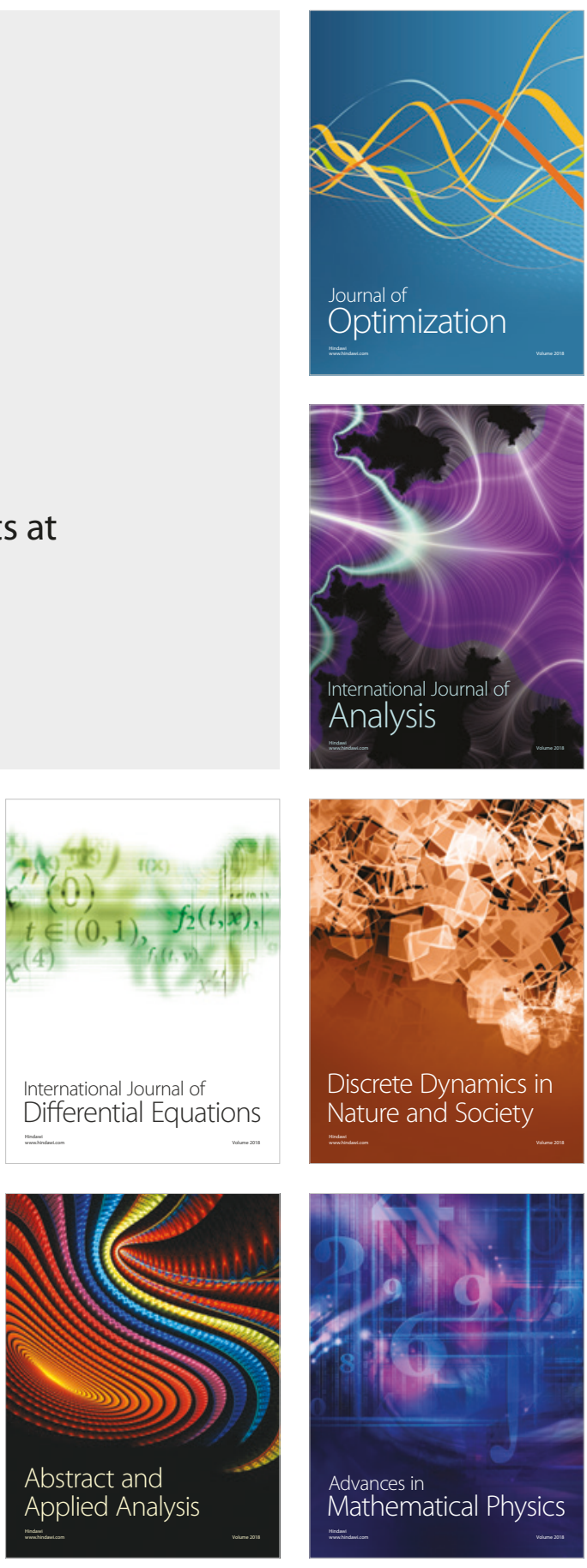